\title{
HYPOTHESIS
}

\section{Neurocardiogenic syncope: a model for SIDS}

\author{
M Ledwidge, G Fox, T Matthews
}

\begin{abstract}
A 51/2 month old male infant who had suffered three acute life threatening episodes was admitted for overnight sleep studies but was found dead after their completion while still in hospital. A necropsy classified the cause of death as sudden infant death syndrome (SIDS). The sleep studies had shown no periods of apnoea $(>20 \mathrm{sec}-$ onds) or bradycardia ( $<90$ beats/min), and a rapid response to nasal occlusion (5 seconds). However, autonomic function during sleep was poor, with reduced heart rate variability (6 beats/min $v$ control 24 beats/min, SD 6.2) and postural hypotension (a 12-14\% fall in resting systolic blood pressure) associated with a fall in heart rate when tilted to a vertical position. Postural hypotension with bradycardia occurs in adults with unexplained syncopal episodes and is called a neurocardiac reflex. It involves poor vasomotor tone with peripheral pooling of blood, a consequent reduction in central venous return and cardiac distension, and in some individuals a neurally mediated bradycardia, as seen in this infant, rather than the expected tachycardia. A progressive bradycardia is the predominant mechanism of death seen in SIDS infants dying on cardiorespiratory monitors at home. This case suggests that a neurocardiac reflex occurs in infants, may have been involved in this infant's death, and deserves further study in the context of SIDS.
\end{abstract}

(Arch Dis Child 1998;78:481-483)

Department of

Paediatrics, Rotunda

Hospital and The

Children's Hospital,

Temple Street, Dublin

1 , Republic of Ireland

$M$ Ledwidge

T Matthews

Department of Paediatrics, Castlebar General Hospital, Castlebar, Co Mayo, Republic of Ireland G Fox

Correspondence to: Professor Matthews.

Accepted 31 December 1997

Keywords: neurocardiac reflex; sudden infant death syndrome

Sudden infant death syndrome (SIDS) is the most common cause of death in infancy, outside the neonatal period, and remains unexplained. ${ }^{1}$ While recently established risk factors (prone sleeping, parental cigarette smoking, and overheating) have allowed the introduction of "reduce the risks" campaigns and have resulted in a welcome decline in SIDS rates in many countries, ${ }^{2-4}$ there remains a gap in understanding how epidemiological risk factors interact with physiological homeostasis to increase the risk of death. Suggested mechanisms of death in SIDS include sleep apnoea, airway occlusion, and failure of the arousal response. ${ }^{5}$ Extensive research has failed to establish a link between SIDS and any of these suggested mechanisms of death, therefore, there is a need to develop alternative mechanisms that can be tested in research studies. It has been suggested that disordered autonomic control may be involved in SIDS. ${ }^{6}$ As part of an ongoing study of autonomic function in infants we report an infant who died of SIDS while in hospital, three hours after overnight cardiorespiratory and autonomic function testing revealed normal respiratory function and abnormal autonomic function.

\section{Case report}

A $5 \frac{1 / 2}{2}$ month old Irish white male infant of unrelated parents was admitted electively to the Rotunda paediatric unit for overnight sleep studies because of a history of apparent life threatening events. The infant was born by a spontaneous vaginal delivery at term after a normal pregnancy. Birth weight was $3030 \mathrm{~g}$. Apgar scores were 9 at one minute and 10 at five minutes, and there were no neonatal or early infancy problems. At 8 weeks old he was found, in the early hours of the morning while sleeping, to be apnoeic, limp, and apparently lifeless, requiring initial shaking and subsequent mouth to mouth resuscitation. Immediately after this episode he was admitted to the local hospital where observation and investigation revealed no abnormality.

He was discharged home after three days on a home apnoea monitor. He had similar episodes at 11 and 15 weeks, occurring at 03:30 and 04:15, each requiring vigorous resuscitation. On hospital admission after each occasion no abnormality was found.
INVESTIGATIONS AND RESULTS

On admission he was well, growing and developing normally, weight $7.68 \mathrm{~kg}$ (50th centile), length $64 \mathrm{~cm}$ (25th centile), and head circumference $42 \mathrm{~cm}$ (50th centile). Physical examination was normal. $\mathrm{He}$ had no fever, was formula feeding normally, and had a resting respiratory rate of 28 breaths/min while quietly asleep. The following investigations were normal: full blood count, urea and electrolytes, arterial blood gasses, routine urine analysis, chest $x$ ray, and electrocardiogram. The remaining tests were conducted during his overnight sleep period in the prone sleeping position from 21:00 to 08:00. Autonomic 
function testing was done as previously described using the heart rate and blood pressure (systolic) response to a change from the horizontal to the upright position during sleep, and the heart rate variability during clinically staged quiet sleep. ${ }^{67}$ An eight hour recording during sleep of respiratory movements and heart rate using a Corometrics 512 neonatal cardiorespiratory monitor (Corometrics Medical Systems, Wallingford, Connecticut, USA) showed no prolonged apnoea ( $>20$ seconds) or bradycardia ( $<90$ beats/min). However, there was a profound reduction in heart rate variability in quiet sleep, with long term heart rate variability of 6 beats $/ \mathrm{min}$ compared with a mean (SD) value of $24(6.2)$ beats/min in a group of healthy 11-13 week old Irish infants. ${ }^{8}$ Long term heart rate variability is the difference between the maximum and the minimum heart rates over 512 consecutive beats, expressed in beats/min, derived from ECG chest electrodes using a peak detecting, instantaneous, digital, event to event cardiotachometer with an accuracy of $\pm 1 \%$.

Blood pressure was measured using an Ohio neonatal blood pressure monitor (Madison, Wisconsin, USA) and an appropriate sized infant cuff on the right upper arm. The values of systolic blood pressure were used in expressing the results. Resting blood pressure was defined as the mean of six readings taken at one minute intervals during sleep immediately before tilt testing. We measured heart rate response over one minute, and blood pressure response at one minute following a change from the horizontal prone sleeping position to the head up vertical position (with the infant's head and trunk at $90^{\circ}$ from the horizontal). The infant was lifted manually into this position and remained asleep during this study. His blood pressure fell by $14 \%$ when in the vertical head up position, and this was associated with a progressive fall in heart rate from 110 to 80 beats $/ \mathrm{min}$. This procedure was repeated twice and yielded a similar result on one occasion (a $12 \%$ fall in blood pressure associated with a fall in resting heart rate from 115 to 95 beats/min) and maintenance of resting blood pressure with no heart rate change on the third test.

The final test was to measure the response time to nasal occlusion. This was assessed during clinically staged quiet sleep by gentle digital occlusion of the orifices of the anterior nares, noting the time taken to arousal as shown by either head movement or the establishment of an oral airway. This infant had a brisk nasal occlusion response time of 5 seconds, compared with an average of 7.8 seconds in similarly tested normal infants. ${ }^{9}$ Following completion of the sleep studies at 08:00, the infant was fed, changed, and placed to sleep in the lateral position at 09:30 attached to a Graseby MR10 monitor (Graseby Medical Co, Watford, UK). He was found at 10:20 by his mother, dead in the prone position with the alarm sounding. A necropsy examination by an experienced paediatric pathologist ascribed the cause of death to SIDS.

\section{Discussion}

This infant was electively admitted for sleep studies because of three previous, severe, sleep related apparent life threatening episodes. $\mathrm{He}$ was studied while sleeping prone and overnight, both being risk factors for SIDS. ${ }^{2}{ }^{3} \mathrm{He}$ was well on admission, with a normal physical and developmental examination and normal vital signs. As measured, respiratory control was normal with no prolonged apnoea and a rapid response to airway occlusion, making sleep apnoea or a deficient response to airway occlusion unlikely causes of death. Autonomic function was abnormal, with a profoundly reduced heart rate variability and a fall in blood pressure associated with progressive bradycardia on upright postural challenge. This response implies poor peripheral vasomotor tone and an inappropriate heart rate response to diminished venous return. ${ }^{10}$

In adults, unexplained syncopal episodes are often associated with poor peripheral vasomotor control, best diagnosed by a head up tilt test that causes hypotension with an associated bradycardia and may reproduce the symptoms. ${ }^{10}$ This has been called neurocardiogenic syncope (or vasovagal syncope). While the precise pathways involved are uncertain, it clearly involves an inappropriate peripheral vasomotor response to a postural challenge, by allowing blood pressure to fall while failing to develop a compensatory tachycardia in the presence of a (presumed) reduction in central venous return. A report of six infants dying from SIDS while attached to a cardiorespiratory monitor at home with a built in memory showed a progressive bradycardia to be the major terminal event in all cases, with continuation of respiratory movements until death. ${ }^{11}$ In neurocardiogenic syncope the essential feature seems to be poor peripheral vasomotor tone, diagnosed by a posturally induced fall in blood pressure associated with a paradoxical, vagally mediated bradycardia secondary to reduced cardiac distension, and in some patients followed by a period of sinus arrest. ${ }^{10}$ In some adult patients a reduction - or withdrawal — of resting sympathetic tone also occurs, causing peripheral, particularly muscle bed, vasodilatation. This further reduces venous return and reinforces the neurally mediated bradycardia. Poor vasomotor tone would be exacerbated by overheating, a known risk factor for SIDS, and the reduction in venous return accentuated by the physiological anaemia, which reaches its nadir at the peak age of occurrence of SIDS. This case suggests that a neurocardiac reflex can occur in infants, may have been involved in this infant's death, and deserves further study both in the context of infant homeostasis and SIDS.

1 Bacon CJ. Cot death after CESDI. Arch Dis Child 1997;76:171-3.

2 Dwyer T, Ponsonby AL. Sudden infant death syndrome: after the "back to sleep" campaign. BMF 1996;313:180-81.

3 Fleming PJ, Blair PS, Bacon C, et al. Environment of infants during sleep and risk of the sudden infant death syndrome: results of 1993-95 case-control study for confidential results of $1993-95$ case-control study for confidential
enquiry into stillbirths and deaths in infancy. BMF 1996;313:191-5.

4 Dwyer T, Ponsonby AL, Blizzard L, Newman NM, Cochrane JA. The contribution of the changes in the 
prevalence of prone sleeping position to the decline in SIDS in Tasmania FAMA 1995;273:783-9.

5 Milner AD, Ruggins N. Sudden infant death syndrome: recent focus on the respiratory system. BMF 1989;298: 689-90.

6 Matthews TG. The autonomic nervous system-a role in sudden infant death syndrome. Arch Dis Child 1992;67: 654-6.

7 Prechtl HFR. The behavioural states of the newborn infant: a review. Brain Res 1974;76:185-212.

8 White MJ. The autonomic nervous system in young infants.
Dublin: University College Dublin, National University of Ireland, 1995:140. [MD thesis.]

Rodenstein DO, Perlmutter N, Stanescu DC. Infants are not obligatory nasal breathers. Am Rev Resp Dis 1985;131: 343-7.

10 Kenny RA, Ingram A, Bayliss J, et al. Head up tilt: a useful test for investigating unexplained syncope. Lancet 1995;i: 1352-5.

11 Meny RG, Carroll JL, Carbone MT, et al. Cardiorespiratory recordings from infants dying suddenly and unexpectedly at home. Pediatrics 1994;93:44-9.

\section{Low birthweight and vision}

The Oxford Register of Early Childhood Impairment was set up in 1984 to collect information about children born in four counties of central England with severe visual impairment, cerebral palsy, or sensorineural deafness. Data have recently been published about children with severe visual impairment born in the four years 1984-87 (Crofts and colleagues; British Fournal of Ophthalmology 1998;82:9-13; also editorial British fournal of Ophthalmology 1998;82:1-2).

There were 132416 live births during the study period and 166 children (1.25/1000 live births) had severe visual impairment (acuity in better eye $6 / 18$ or less) by the age of 5 years. The rate was $26 / 1000$ for 1118 children with birthweight below $1500 \mathrm{~g}, 3.6 / 1000$ at birthweights $1500-2499 \mathrm{~g}, 1.0 / 1000$ at $2500-3499 \mathrm{~g}$, and $0.7 / 1000$ at $3500 \mathrm{~g}$ and over.

Children with very low birthweight constituted $0.8 \%$ of all live births and $17.5 \%$ of those with severe visual impairment. The visual impairment was considered to be of prenatal or perinatal origin in $84 \%$ of all visually impaired children, and in $90 \%$ of those with very low birthweight, but it was considered perinatal in most very low birthweight children and prenatal in most of those with higher birthweight.

The most common cause was cortical visual impairment, which occurred in 60 children (36\%) and was thought to be of prenatal origin in 27 , perinatal in 23 , and postnatal in 10 . Only nine children had retinopathy of prematurity. Other causes were optic atrophy (21), cataract (16), congenital nystagmus (10), and several less common diagnoses. Associated motor, sensory, or developmental impairments were present in $72 \%$ of the very low birthweight visually impaired children and $54 \%$ of those who weighed more than $2500 \mathrm{~g}$ at birth.

Cerebral ischaemia is a more important cause of visual impairment in very low birthweight babies than is retinopathy of prematurity (ROP), at least in the UK. Fielder, in his editorial, however, points out that ROP is increasing in middle income countries such as those in Latin America and eastern Europe where neonatal care is such that more preterm babies are surviving but more are getting ROP. Nevertheless, two thirds of the children with severe visual impairment in this study weighed more than $2500 \mathrm{~g}$ at birth. Attempts to reduce the burden of visual impairment in children in developed countries will need to address the problems of genetic and other prenatal causes, and cerebral ischaemia. 